Meta

Journal des tradlucteurs

Translators' Journal

\title{
Le traducteur professionnel et les dictionnaires techniques
}

\section{Henri Van Hoof}

Volume 16, numéro 4, décembre 1971

URI : https://id.erudit.org/iderudit/003080ar

DOI : https://doi.org/10.7202/003080ar

Aller au sommaire du numéro

Éditeur(s)

Les Presses de l'Université de Montréal

ISSN

0026-0452 (imprimé)

1492-1421 (numérique)

Découvrir la revue

Citer cet article

Van Hoof, H. (1971). Le traducteur professionnel et les dictionnaires techniques. Meta, 16(4), 211-215. https://doi.org/10.7202/003080ar d'utilisation que vous pouvez consulter en ligne.

https://apropos.erudit.org/fr/usagers/politique-dutilisation/ 


\section{le Traducteur professionnel \\ et les Dictionnaires techniques}

0. Le traducteur professionnel, s'il n'est déjà pas méfiant de nature, est bien vite rendu circonspect par les dictionnaires techniques. L'expérience lui apprend, en effet, que ces derniers lui fournissent rarement la solution «sur mesure » à ses problèmes. Or, qu'attend le traducteur spécialisé d'un dictionnaire technique? 0.1 . Avant de répondre à cette question, il n'est peut-être pas inutile de jeter un regard sur la méthode du traducteur. Placé devant un texte technique ou scientifique dont le domaine ne lui est point familier, il va commencer par se documenter en consultant les encyclopédies générales d'abord, les encyclopédies spéciales ensuite, les traités, manuels et autres ouvrages de référence enfin. Dans la mesure où elle est possible, cette consultation se fera dans la langue de départ et dans la langue d'arrivée, la comparaison de textes homologues permettant de faire apparaître déjà certaines équivalences terminologiques et de résoudre avant la lettre quelques-uns des problèmes de traduction qui pourraient se poser.

0.2. Le texte étant ainsi identifié et son message compris, seuls des obstacles d'ordre terminologique pourront encore arrêter' le traducteur. Ces obstacles pourront être de nature très variée, selon qu'il s'agit de néologismes, d'éponymes, de termes normalisés ou appartenant au lexique courant d'une activité spécifique, de jargon technique propre à une discipline, à une région, à une entreprise déterminées. Et l'on voit ainsi se préciser le type de renseignements que le traducteur s'attend à trouver dans un dictionnaire technique bien fait. Il n'y cherchera pas drague, mais peut-être bien drague suceuse; il n'y cherchera peut-être pas gastrectomie s'il est spécialisé en médecine, mais vérifiera quand même gastrectomie à la Bilroth $I$; et il y cherchera certainement (mais en vain) le chapeau mexicain bien connu des chimistes de laboratoire et qui désigne un mélange de fusion alcalin aux carbonates que les spécialistes anglophones appellent tout simplement carbonate fusion mixture.

0.3. En bref, l'examen des exigences du traducteur professionnel à l'égard des dictionnaires techniques revient à poser les critères de sélection, de discrimination 
et de systématique auxquels ces ouvrages devraient répondre. C'est ce que nous allons nous efforcer de faire en nous référant à une série de dictionnaires récents qui intéressent les langues française, néerlandaise, anglaise et allemande.

\section{CRITÈRES DE SÉLECTION}

1.1. On serait tenté de considérer comme acquis le souci du lexicographe de couvrir au maximum la terminologie du domaine étudié et de s'en tenir exclusivement à cette terminologie. La pratique montre que nous sommes souvent loin du compte. Le Dictionary of Science and Technology (En-De) de Dorian prétend explorer la terminologie de 130 champs d'activité, mais reste superficiel dans la plupart ; le Technical and Engineering Dictionary (En-De/De-En) de De Vries, même dans sa dernière édition (1967), contient trop de matière inutile. Ce dernier travers se retrouve dans des dictionnaires jouissant par ailleurs d'une bonne réputation d'ensemble. On est assez surpris de rencontrer des termes comme chauffage central, chauffeur, engrais, allocation familiale, assistante sociale, etc., dans le Dictionnaire commercial et financier de Servotte, où l'on cherche en vain carte perforée et comptométrice; dans le Dictionnaire technique de la construction ( Fr-Ne/Ne-Fr) de Venstermans, on tombe en arrêt devant une expression comme supprimer un embouteillage, mais il manque des termes comme silicone, hydrophobe, inhibiteur de prise, drague à berme, drague à désagrégateur, etc.; dans le Frans-Nederlands Technisch Woordenboek de Voutquenne, on tombe sur des mots comme prime, contrat et autres verbaliser, mais on note l'absence de termes comme composition mécanique, simili, détouré et du sens technique de certains mots du lexique général (réserver, finition, etc.).

1.2. Ce dernier point soulève d'ailleurs un problème assez délicat. Certains auteurs, en effet, pour répondre au fréquent reproche de padding, ont pris parfois des décisions extrêmes. C'est le cas de Lejeune et Bunjes qui, dans leur excellent Deutsch-englisches Wörterbuch für Aerzte, ont éliminé tous les mots dont les acceptions n'étaient pas exclusivement médicales. Cette attitude, parfaitement défendable si l'on tient compte du développement rapide et multiforme de la terminologie médicale, est toutefois discutable dans la mesure où elle risque d'exclure des termes d'apparence non médicale qui prennent une signification bien définie dans un contexte médical (nous pensons à l'anglais complaint, case history, patent, etc., au français urgent, etc.).

1.3. Nombreux sont les dictionnaires, même bien faits, qui sous leur titre technique général, se limitent en fait à une série de domaines seulement. Les termes du Technisch Engels Woordenboek de Jansonius proviennent surtout de la métallurgie, de la mécanique, des machines-outils, des moteurs, de l'électro-technique. Le German-English Technical Dictionary de Leidecker est particulièrement bien fourni en termes d'aéronautique et d'astronautique, de photographie aérienne et de topographie, de ballistique et de fusées. Le Technisches Wörterbuch (De-Fr/Fr-De) de Lehmann couvre principalement la chimie, l'exploitation minière, la fonderie, la construction mécanique. Le Dictionary of Technical Terms and Phrases (Fr-En/En-Fr) de Kettridge se montre très complet surtout dans le génie civil, 
la construction mécanique, les industries extractives, la géologie, la minéralogie et la métallurgie.

1.4. Une des grandes lacunes, enfin, pour laquelle les dictionnaires se voient souvent critiqués est celle de la phraséologie. Entendons par là des locutions, des tournures verbales, adverbiales ou autres propres à un domaine terminologique particulier et qui, même si leurs termes paraissent clairs, appellent une traduction qui peut ne l'être pas pour autant. Des tours simples tels que à hauteur de, au droit de, au champ de, dans l'axe de, etc., des locutions verbales comme porter au bouillon, amener au volume, reprendre (un résidu) par de l'eau, etc., peuvent créer des difficultés selon la langue d'arrivée. Or, c'est un champ d'investigation que la plupart des lexicographes ignorent ou ne font qu'effleurer. On n'en remarquera que d'autant mieux un Dictionary of Commercial and Financial Terms and Phrases (Fr-En/En-Fr) de Kettridge, ou un Dictionnaire agricole (De-En-Fr-El) de Haensch et Haberkamp, ou un Dictionnaire commercial et financier (déjà cité) de Servotte, ou un Dictionary of Civil Engineering and Construction, Machinery and Equipment (Fr-En-De) de Bucksch, qui tous ont fait un gros effort dans le sens souhaité par les traducteurs. Et peut-être n'est-ce pas simple coïncidence si leurs auteurs ont eu une longue pratique de la traduction.

\section{CRITÈRES DE DISCRIMINATION}

2.1. En admettant que la sélection retenue par le lexicographe soit représentative et pertinente, il n'en résulte pas pour autant que l'exploitation qui en est faite répond aux desiderata du traducteur. Si l'auteur s'est contenté de donner les différentes traductions de ses termes sans en préciser les acceptions spécifiques ou les champs d'application, la portée pratique de son ouvrage sera fort compromise. De plus, s'il aligne les différentes acceptions en traduction comme des équivalents apparemment synonymes, le risque d'équivoque et de contresens devient grand pour l'utilisateur. Pour ne comparer que deux ouvrages récents parus en Belgique, il est clair que le Dictionnaire technique de la construction de Venstermans pêche par manque de discrimination et sur le plan de la synonymie et sur le plan des aires d'application, tandis que le Technisch Woordenboek de Voutquenne, dont le contingent de termes relevant de la construction n'est pas négligeable, distingue très bien les domaines d'utilisation des traductions données comme équivalents. Toujours dans la même discipline, le Dictionnaire pour les travaux publics (déjà cité) de Bucksch fait preuve d'un souci très poussé de la synonymie.

2.2. Les solutions données à ce problème de la synonymie par les lexicographes qui lui ont porté quelque attention sont fort variées. Si l'on examine la façon dont les mots sont traités dans le Dictionnaire commercial et financier de Servotte, par exemple, on peut regretter parfois la synonymie assez réduite. Prenons le terme courant d'affaires; dans la colonne du néerlandais, on trouve uniquement omzet, alors que selon le cas on peut devoir traduire par handel, kooplust, levendigheid, etc. Ainsi : un bon courant d'affaires : een levendige handel; le blé a eu le plus vif courant d'affaires : het graan werd het meest verhandeld. Prenons encore 
l'expression entamer son capital ; elle est rendue en anglais par to ancroach upon one's capital, mais il faudrait y ajouter to eat into, to dip into, to draw on one's capital, qui sont d'utilisation plus courante dans les presses économique et financière. Dans l'introduction de leur Wörterbuch für Aerzte, Lejeune et Bunjes. nous promettent de donner les diverses traductions anglaises plus ou moins. synonymes des mots allemands. Cette bonne intention est souvent démentie dans les faits : on ne trouve ni râle ni rattling à côté de rhonchus (pour l'allemand Giemen); on ne trouve le synonyme air passages ni à la rubrique Atemwege (respiratory system, -passage, -tract) ni à la rubrique Luftwege (respiratory tract). Le Wörterbuch der industriellen Technik (De-En/ De-Fr/ Fr-De) de Ernst a essayé de fournir une solution originale en faisant suivre chaque mot clé, avant ses traductions, de synonymes éventuels dans la langue de départ. Par exemple : résistance, force portante, limite de charge $=$ Tragfähigkeit ; réajuster, rajuster, régler de nouveau, parfaire le réglage $=$ nachregeln. Cette méthode, si elle ne résout pas entièrement le problème des champs d'application, offre du moins l'avantage de s'appuyer sur la compétence de l'utilisateur dans la langue de départ.

2.3. Nous ne citerons que pour mémoire les discriminations à faire entre certains usages nationaux ou régionaux. $\mathrm{La}$ plupart des dictionnaires techniques récents font la distinction entre l'usage GB et l'usage US pour l'anglais, certains le font entre les usages $\mathrm{F}, \mathrm{B}, \mathrm{Ca}$ et $\mathrm{CH}$ pour le français, entre les usages $\mathrm{NN}$ et $\mathrm{ZN}$ pour le néerlandais.

\section{CRITÈRES DE SYSTÉMATIQUE}

3.1. Au cours des quinze dernières années, de nombreuses études ont été publiées sur la nécessité de rénover les principes de la compilation lexicographique. Nous n'entrerons pas dans le détail des multiples tendances de la lexicographie moderne, mais voudrions simplement attirer l'attention sur quatre ouvrages qui s'en sont inspirés et qui, à des titres divers, se rapprochent de l'idéal souhaité par le traducteur professionnel.

3.2. Nous avons cité déjà le Dictionnaire agricole de Haensch et Haberkamp. L'originalité de cet ouvrage réside dans sa présentation systématique, c'est-à-dire qu'il repose sur un classement rationnel des matières, groupées ici en quatorze chapitres. À ceux qui reprocheraient à cette méthode d'exiger une connaissance préalable $\mathrm{du}$ sujet sous peine d'augmenter les difficultés de consultation, les auteurs ont répondu par l'adjonction d'un index alphabétique complet pour chacune des langues traitées (Fr-De-En-El), faisant ainsi de cette terminologie systématique un dictionnaire technique d'un maniement très facile. C'est d'ailleurs cette même conception qui a fait, pensons-nous, le succès de la collection Wirtschaftssprachen publiées aux Éditions Max Hueber.

3.3. Dans certains cas, les auteurs ont choisi de rédiger un texte suivi et cohérent qui permet, mieux qu'un dictionnaire, de préciser le sens des termes et expressions techniques. Les Terminologies chimiques (Fr-En/Fr-De/De-En) de Fromherz et King en sont un bel exemple. Les textes français et anglais, français et allemand 
ou anglais et allemand sont présentés en regard l'un de l'autre, de manière à faciliter la comparaison. Le traducteur peut voir ainsi les termes spécialisés évoluer dans leur contexte habituel, où ils sont imprimés en italique la première fois qu'ils interviennent. Tous ces termes sont ensuite repris dans des index alphabétiques, compléments indispensables de ce type d'ouvrage.

3.4. D'autres ouvrages limitent la classification systématique à certaines matières réunies sous un même mot clé. Cette disposition particulière, d'une très grande utilité pratique, caractérise notamment les divers volumes du Wörterbuch für Ingenieurbau und Baumaschinen de Bucksch. Ainsi, à grands barrages on ne trouve pas moins de 18 pages de vocables se rapportant à leur construction; de même 8 pages à construction de tunnels, 5 pages à eaux usées, 3 pages à bétonnières, etc. Elle se retrouve aussi dans la série des Kunststoff-Technische Wörterbücher de Wittfoht. À côté d'une première partie, constituant le dictionnaire proprement dit, qui donne dans l'ordre alphabétique tous les termes, composés et expressions des différentes branches de l'industrie des matières plastiques, chaque volume contient une partie encyclopédique où sont rassemblés, dans des rubriques collectives $: a$ ) les procédés de moulage, de formage et de profilage, $b$ ) les presses et les moules, $c$ ) les procédés de transformation et d'usinage, $d$ ) les mélangeurs et accessoires. Toutes ces rubriques sont richement illustrées. Il en est de même d'ailleurs de la partie alphabétique, où d'innombrables schémas et dessins avec légendes bilingues aident à visualiser les concepts à traduire; complétant des définitions d'une précision et d'une concision exemplaires, ils contribuent à faire du Wittfoht plus qu'un dictionnaire technique traditionnel - une encyclopédie bilingue basée sur une documentation énorme, dépouillée avec pertinence et présentée avec clarté.

3.5 Revenons pour terminer, au Wörterbuch für Aerzte de Lejeune et Bunjes. Issu de la collaboration entre un médecin et un traducteur médical et lexicographe, il est parmi les rares à tenir compte délibérément de l'optique du traducteur en accordant une importance inhabituelle aux pièges de traduction les plus courants, aux faux amis, à certaines équivoques terminologiques, à certaines difficultés pratiques de traduction, bref, à toute une série de petits problèmes qui sortent du cadre purement lexicographique, mais qui sont le lot quotidien du traducteur professionnel et que ce dernier est dès lors heureux de voir traiter dans un ouvrage de ce genre.

4. S'il nous fallait conclure, nous poserions que le traducteur professionnel a très certainement un rôle à jouer dans l'orientation future et le perfectionnement des dictionnaires techniques - un rôle de critique, un rôle de consultation, un rôle de participation. Le travail lexicographique proprement dit devra toutefois rester aux mains du spécialiste, afin que l'avenir nous épargne ces lexiques et glossaires obtenus par transcription pure et simple de fichiers personnels qui, même fournis par des traducteurs expérimentés, s'avéreront inutilisables s'ils n'ont pas été régis par des principes lexicographiques bien définis.

HeNRI VAN HoOF 